\title{
DEVELOPMENT OF VOCAL TEMPORAL PARAMETERS IN DISTANTLY RELATED VOCAL LEARNERS, BIRDS AND HUMANS
}

\author{
MIKI TAKAHASI ${ }^{* 1,2}$, KAZUO OKANOYA $^{3}$, and REIKO MAZUKA ${ }^{1,4}$ \\ *Corresponding Author: mtakahas@brain.riken.jp \\ ${ }^{1}$ Lab. for Language Development, RIKEN BSI, Japan \\ ${ }^{2}$ JSPS Research Fellow, Japan \\ ${ }^{3}$ Department of Life Sciences, Graduate School of Arts and Sciences, the \\ University of Tokyo, Japan \\ ${ }^{4}$ Department of Psychology and Neuroscience, Duke University, Durham NC, \\ USA
}

Young animals which are immature and immobile produce vocalizations such as isolation calls or cries to elicit care from adults. A common feature of these vocalizations is that the several vocal elements make a sequential vocalization. Sequential vocalizations accompany constant control of the air flow (reviewed in Doupe \& Kuhl, 1999; Riede \& Goller, 2010). Although sequential vocalizations eliciting care may largely be innate and involuntarily controlled for many animals, some animals, such as songbirds, are capable of learning new sequential vocalizations through social experiences. These vocal learners have been found to have a direct projection from cerebral cortex to vocal-respiratory center in medulla (Jurgens, 2002). This direct projection enables voluntary respiration and vocalization during vocal learning. Veit et al. (2011) showed juvenile songbirds learn the link between respiration and vocalization. In human infants, respiratory rate in crying and movements of lung and abdomen change through development (Boliek, Hixon, Watson, \& Morgan, 1996; Wilder \& Baken, 1974). However, it is not known yet when the shift from involuntary to voluntary respiration occurs in vocal learning process. The present paper analyzed the early vocalizations in human infants and Bengalese finches, one of songbird species, to address this question.

We focused on the developmental changes in 3 temporal parameters in sequential vocalizations: Note Duration (ND), Inter-Onset-Interval (IOI), and 
Inter-Note-Interval, silent interval between two adjacent notes (INI). To produce sequential vocalizations, respiration must be controlled. Thus, the analyses of temporal parameters for sequential vocalizations may reveal the timing of the developmental shift from the involuntary to voluntary respiration control. We used the longitudinally recorded vocalization from 4 infants and 6 young finches. In infants data, crying at 0 month were also analyzed to compare with the sequential vocalizations.

For human infants, the distribution patterns of temporal parameters in cries differ from those in spontaneous sequential vocalizations as shown in Fig 1. The mean duration of ND gradually increased up to 5 months of age, when it became close INI. This pattern was similar to neonates' cries. In Bengalese finches, INI gradually declined up to 70 days, although merging of ND and INI occurred, too. However, combining a specific ND with a specific INI appeared through development in Bengalese finches. IOI kept its distribution pattern in humans. In general, the vocalizations of 5-month-olds are known to become highly variable as vocal playing. Based on these data, involuntary/voluntary switching might occur at 5 months of age in humans. In Bengalese finches, voluntary respiration control may enable to stabilize sequential vocalization at around 70 days.
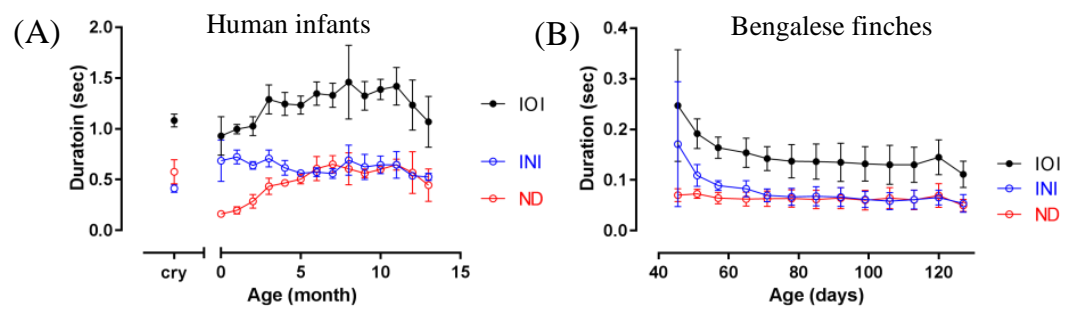

Figure 1. Developmental change of mean durations of three temporal parameters.

\section{Acknowledgements}

This work was supported in part by JSPS Grant-in-Aid for JSPS Research Fellow (16J40180) to MT, Scientific Research S (16H06319) to RM, and MEXT Grant-in-Aid for Scientific Research on Innovative Areas in \#4903 (Cocreative Language Evolution, 17H06380) to RM. 


\section{References}

Boliek, C. A., Hixon, T. J., Watson, P. J., \& Morgan, W. J. (1996). Vocalization and breathing during the first year of life. Journal of Voice, 10(1), 1-22.

Doupe, A. J., \& Kuhl, P. K. (1999). BIRDSONG AND HUMAN SPEECH: Common Themes and Mechanisms. Annual Review of Neuroscience, 22(1), 567-631.

Jurgens, U. (2002). Neural pathways underlying vocal control, 26.

Riede, T., \& Goller, F. (2010). Peripheral mechanisms for vocal production in birds - differences and similarities to human speech and singing. Brain and Language, 115(1), 69-80.

Veit, L., Aronov, D., \& Fee, M. S. (2011). Learning to breathe and sing: development of respiratory-vocal coordination in young songbirds. Journal of Neurophysiology, 106(4), 1747-1765.

Wilder, C. N., \& Baken, R. J. (1974). Respiratory patterns in infant cry. Human Communication, (Winter), 18-34. 